\title{
Carnets
}

Revue électronique d'études françaises de l'APEF

Deuxième série - 8 | 2016

Du Français en cause aux causes du français

\section{Le français professionnel dans la diffusion du français au Portugal}

Le cas de l'école supérieure de gestion, hôtellerie et tourisme de

l'université de l'Algarve

\section{Catherine Pasquier et Idília dos Santos}

\section{(2) OpenEdition}

Journals

Édition électronique

URL : http://journals.openedition.org/carnets/1786

DOI : $10.4000 /$ carnets. 1786

ISSN : 1646-7698

Éditeur

APEF

Référence électronique

Catherine Pasquier et Idília dos Santos, «Le français professionnel dans la diffusion du français au Portugal », Carnets [En ligne], Deuxième série - 8 | 2016, mis en ligne le 30 novembre 2016, consulté le 19 avril 2019. URL : http://journals.openedition.org/carnets/1786 ; DOI : 10.4000/carnets.1786

Ce document a été généré automatiquement le 19 avril 2019

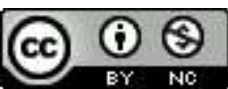

Carnets est mis à disposition selon les termes de la licence Creative Commons - Atribution - Pas d'utilisation commerciale 4.0 International. 


\title{
Le français professionnel dans la diffusion du français au Portugal
}

Le cas de l'école supérieure de gestion, hôtellerie et tourisme de l'université de l'Algarve

\author{
Catherine Pasquier et Idília dos Santos
}

1 L'enseignement $d u$ français langue étrangère semble connaître actuellement des problèmes relevant d'ordres divers: le désengagement financier des gouvernements français dans la diffusion de la langue française dans le monde, le déclin économique de la France, la sphère anglo-saxonne qui promeut l'anglais au statut de langue de communication internationale. Mais si l'anglais est vital pour l'exercice des professions dans un monde globalisé, il ne distingue plus les candidats à l'emploi auxquels on demande des profils linguistiques diversifiés.

Dans le cadre de ce numéro de Carnets sur les problématiques de la diffusion du français, nous souhaitons, au contraire, à travers notre témoignage de 14 années d'enseignement $\mathrm{du}$ français en licence professionnelle, nuancer fortement cette vision de crise de l'enseignement de la langue française qui résulte, en partie, du manque d'adaptation à l'évolution des sociétés contemporaines. En effet, parallèlement aux motivations reposant sur la communication avec des francophones et l'accès aux valeurs universelles de la culture des arts et des lettres, de nouvelles motivations pour l'apprentissage d'une langue étrangère, de nouveaux besoins émergent, s'étendent ou se massifient et sont autant d'opportunités pour la diffusion de la langue française.

Dans le contexte de notre participation à cette diffusion, nous explorerons, tout d'abord, la demande de formations en français non généraliste dans le cadre d'une économie mondialisée, en mettant en relief leur caractère spécifique et évolutif et le besoin d'offres de formations contextualisées, adéquates au développement de compétences en langues étrangères, créatrices de valeur, au sens économique du terme, pour l'individu, l'entreprise, le territoire.

4 Puis nous présenterons le cas de l'École Supérieure de Gestion, Hôtellerie et Tourisme de l'Université de l'Algarve (désormais ESGHT) : les caractéristiques de ses étudiants, et des formations ainsi que les actions d'adaptation des méthodes et des contenus aux 
évolutions socio-professionnelles afin de répondre aux besoins des apprenants et à ceux des nouvelles activités d'interactions langagières au travail. De fait, nous travaillons dans un contexte économique précis et nous acceptons que l'économie et l'éducation interagissent et conditionnent mutuellement la réussite de leurs objectifs. A titre d'exemple, nous montrerons d'ailleurs comment des mesures de politique économique et fiscale, sans rapport direct avec les politiques linguistiques, peuvent contribuer à une revalorisation la langue française au Portugal.

\section{Comprendre les demandes}

5 Pour situer ces demandes, il nous parait nécessaire de mentionner l'évolution des relations entre langue et travail car les pratiques linguistiques au travail ont changé. Depuis le modèle de Taylor qui séparait la fonction de conception de celle d'exécution manuelle, d'où la parole était bannie, l'évolution progressive de l'activité économique avec l'explosion des industries de services et de la culture managériale, où désormais tout le monde est appelé à communiquer, a fait du langage une composante essentielle du travail et de nombreuses tâches ne sont possibles que par l'intermédiaire du langage. Qu'il accompagne le geste ou qu'il soit une activité à part entière, le langage est devenu un outil de production qui a changé l'exercice des professions « le faire revient de plus en plus à $d u$ dire » (Mourlhon-Dallies, 2008), et ce dire se déverse désormais sous diverses formes sur une multitude de supports et écrans.

6 Par ailleurs, ces dires traversent et transforment les frontières linguistiques. Les mobilités et l'internationalisation des productions et des échanges engendrent des pratiques multilingues et multicanales au travail. Pour répondre à ces besoins de communication multilingues, un véritable marché de formations en langues étrangères a vu le jour. Si les débouchés professionnels spécifiques en langues restent limités, en revanche, associée à d'autres compétences techniques comme le droit, le commerce, le tourisme, la communication ou la technologie, la connaissance effective de langues étrangères, si elle est efficace, est valorisée en tant que compétence opérationnelle rentable sur le marché du travail (Bensfia, Mabrour et Mgharfaoui, 2013).

7 Cette augmentation des activités langagières au travail et ces besoins de compétences spécifiques ne sont pas sans conséquence sur la diffusion du français. Les responsables des politiques et formations linguistiques doivent pouvoir y répondre en élaborant des formations connectées aux besoins évolutifs de cette réalité où les quatre habiletés du Cadre Européen pour l'Enseignement des Langues s'entrecroisent.

8 A l'échelle de notre institution, il s'agit de comprendre les besoins des entreprises et des secteurs d'activités qui font vivre sa zone d'implantation car une des missions d'une école supérieure polytechnique est de servir et satisfaire les besoins du marché du travail et du territoire où elle est implantée. Des études (Grin, 2009 ; ALLC, 1994 ; ELAN, 2004) montrent l'importance économique des langues dans l'activité professionnelle et dans la création de valeur ajoutée. Plus les compétences en langues répondent aux attentes et aux besoins du marché, plus elles sont économiquement rentables. Ainsi, pour construire des compétences linguistiques et professionnelles adéquates et en accord avec la mission de notre institution, il nous faut comprendre les changements de la demande des secteurs d'activités et de nos publics. 
Pour les comprendre, il nous faut également nous placer à l'échelle de l'apprenant et du contexte d'enseignement. Pour un individu, il existe énormément de raisons d'apprendre une langue étrangère*. Dans une école supérieure professionnelle, outre l'aspect identitaire, affectif ou familial, ce choix est fonction de son utilité car, à terme, l'acquisition de langues étrangères doit être avantageuse du point de vue professionnel.

De fait, le motif principal de nos étudiants étant le travail, les langues sont considérées comme un moyen d'accès à l'emploi. Outre l'obtention de leurs diplômes, les étudiants cherchent à se doter de compétences nécessaires à leur insertion professionnelle. Ils cherchent une formation linguistique et une valorisation professionnelle. Or pour être valorisée, selon la logique économique, l'acquisition d'une langue doit être, à terme, rentabilisée. Pour donner envie de français, il faut donc qu'en amont, la langue française et la francophonie représentent une occasion de travail et de projets professionnels comme un emploi, un stage, un échange ou une expatriation. Au vu de cette recherche de qualification et de compétences utiles au travail, une formation déconnectée de la réalité professionnelle de leur cursus n'aura pas la faveur de nos publics et ne contribuerait ni à leurs besoins de formation ni à la diffusion du français.

\section{Évolution des formations en langues non généralistes}

11 La prise de conscience de la valeur, pour l'individu, l'entreprise et la région, d'employer des personnes maîtrisant plusieurs langues étrangères dans leur secteur d'activité se traduit par une évolution des formations en français. Cette offre se renouvelle et se spécialise autour d'un rapprochement entre la sphère de l'enseignement en langues et celle du travail. D'une part, les formations de type Français sur objectif(s) spécifique(s) (FOS) qui répondent aux exigences contemporaines des marchés sont en demande. D'autre part pour s'adapter à ces exigences contemporaines, des pratiques plurielles se développent au niveau mondial et, en réponse, la didactique du français non généraliste travaille de nouveaux concepts et positionnements (Mourlhon-Dallies, 2008).

Historiquement, la réflexion scientifique portant sur l'enseignement des langues à finalité pratique et professionnelle s'était amorcée à partir des années 60 . Au contraire du français généraliste qui présente une longue et prestigieuse tradition et une évolution à partir d'une approche centrée sur la grammaire, puis sur le lexique, ensuite sur les textes et la culture (Puren, 2005), l'enseignement du français non généraliste, peu valorisé et souvent effectué par des écoles de langues privées et des enseignants aux statuts précaires, a évolué à partir de pratiques de formation centrées sur le lexique et la terminologie. Ensuite elle s'est concentrée sur le développement de compétences de lecture de textes spécialisés, et par la suite, a donné priorité aux compétences liées à la production écrite, puis orale selon les besoins spécifiques des apprenants (MourlhonDallies, $2008: 14$ ).

Dans les années 2000 a resurgi un intérêt pour les spécificités, la variété et la richesse des méthodologies d'enseignement du français non généraliste et une réflexion théorique s'est amorcée en s'appuyant sur la stabilité de certaines dénominations. Désormais, la distinction entre le FOS, relevant de demandes de formation spécifique et le français de spécialité, relevant d'offres plus transversales, proposée par Mangiante et Parpette (2004) est généralement acceptée. Cette réflexion a participé à la création du nouveau concept de «Français Langue Professionnelle » proposée par Mourlhon-Dallies (2008) qui met au 
cœur des dispositifs d'enseignement la logique des métiers. En 2011, Mangiante et Parpette ont défini le concept de «Français sur Objectifs Universitaires» (Mangiante et Parpette : 2011) en structurant la réflexion sur la construction de formations nécessaires à l'insertion et à la réussite des étudiants universitaires. Finalement le dernier né est le français comme compétence professionnelle, issu du croisement entre la méthodologie actionnelle du FLE et celles utilisées dans la formation professionnelle (De Ferrari, 2011). Il concerne en premier lieu les publics de migrants en formation professionnelle pour lesquels la langue occupe une place primordiale puisqu'elle conditionne les autres apprentissages.

14 La didactique du français non généraliste propose donc de nombreuses appellations, parfois confondues à cause de leurs de chevauchements conceptuels et elle utilise ces dénominations pour spécifier différents types de formations. Toutes ont en commun de proposer une formation spécifique au domaine de spécialisation mais leurs objectifs diffèrent selon leur degré de spécificité et de spécialisation par rapport à des secteurs, à des métiers ou postes de travail spécifiques (Sowa : 2014). Le FOS est parfois proposé dans l'éducation supérieure en tant qu'appellation fédératrice qui vise à prendre en charge » le lien entre langage et action dans les champs des pratiques langagières professionnelles et universitaires » (Mangiante et Richier, $2014: 4$ ).

S'il convient de penser l'offre de formations selon ces vastes changements intervenus dans le monde du travail et des didactiques des langues, il s'agit également, au vu de la valorisation des langues et cultures dans l'économie, d'envisager l'application et l'adaptation des formations aux besoins précis et évolutifs du marché de l'emploi.

\section{Adapter et construire l'offre}

16 Notre réflexion sur l'adaptation de notre offre de formations s'est centrée autour de deux axes que nous avons structurés sous forme d'objectifs visant l'adéquation de l'enseignement aux compétences recherchées: le rapprochement de la langue et de la pratique professionnelle et le rapprochement de l'enseignement de la langue de la formation technique et professionnelle.

Le rapprochement de la langue et de la pratique professionnelle s'est effectué par le déplacement de l'objet de notre démarche. Contrairement au français généraliste dont l'enseignent est centré sur la langue envisagée comme moyen d'accès à la culture littéraire et artistique, le FLP engage une réflexion sur l'activité et la communication au travail. Savoir communiquer et agir professionnellement, ce n'est pas seulement maitriser la grammaire, le lexique ou la phonologie, c'est maitriser également la globalité de l'interaction selon la logique du secteur professionnel. De fait, un énoncé peut être parfaitement correct mais être inacceptable du point de vue du secteur professionnel. " Avant même de parler correctement, encore faut-il parler avec «à propos » c'est-à-dire à la bonne personne, au bon moment, en accord avec le cadre juridique et déontologique de son poste de travail» (Mourlhon-Dallies, 2008: 100). L'exercice d'une profession demande non seulement de maitriser le linguistique mais également de comprendre ce qui est attendu comme "parole, attitudes, réactions, initiatives» (Mourlhon-Dallies, 2008 : 102). Nos objectifs ne peuvent donc pas être strictement linguistiques car outre le verbal, cet "agir" professionnel mobilise le culturel, le technique, le juridique, le comportemental (Mourlhon-Dallies, $2008: 314$ ). 

le sens et la logique du secteur professionnel pour en déduire les compétences à travailler avec les étudiants. Le sens est entendu ici au sens de la linguistique cognitive (Fauconnier et Turner, 2008; Fuchs, 2008; Evans, 2014), non comme sens différentiel mais comme conceptualisation de l'expérience et de l'interaction avec l'environnement, mettant en œuvre les connaissances linguistiques, au sens saussurien du terme, mais aussi toutes les connaissances encyclopédiques nécessaires à la communication. Travailler sur des secteurs de spécialité fait prendre conscience de la primauté du sens dans les interactions humaines.

Ainsi, l'objectif est de faire prendre conscience aux étudiants explicitement de ce qui est prototypique du domaine et de ce qui ne l'est pas comme les types de textes et les agencements spécifiques de formes discursives; des différences de conceptualisation professionnelle et non professionnelle; et de ce qui est transférable entre ces deux domaines, entre la langue maternelle et la langue française et également entre plusieurs disciplines (Causa, 2014 ; Vollmer, 2009). La reconnaissance de ces opérations cognitives générales - transfert, changement de perspective, catégorisation - et leur mise en discours (Boers \& Lindstromberg, 2006 ; De Rycker, \& De Knop, 2015) offre des pistes aux didacticiens pour également développer des méthodes d'enseignement des langues/ compétences professionnelles.

Notre deuxième axe de réflexion porte sur le rapprochement de l'enseignement des langues et la formation technique et professionnelle. Que se passe-t-il quand on passe d'une pratique d'enseignement centrée sur le processus d'apprentissage d'une langue, tout à fait légitime pour des publics de futurs enseignants, à une pratique centrée sur son contexte d'utilisation? Les besoins et donc les objectifs changent. Ce n'est plus seulement la langue qui est objet d'enseignement avec ses dispositifs organisés autour des actes de paroles, des catégories sémantico-grammaticales ou lexicales. Il ne s'agit plus non plus seulement de traiter des textes et des informations comme au temps du français langue de spécialité.

21 L'objectif est d'assurer un enseignement linguistique intégré à une formation de spécialité permettant d'accomplir des actions professionnelles dans toutes leurs dimensions puis de les évaluer en tant que telles. Et pour répondre à ces nouveaux objectifs, il importe de proposer une formation en français permettant d'accomplir des tâches/actions professionnelles du domaine de spécialité grâce à une démarche pédagogique intégrée langue/discipline où la langue est à la fois l'objet et le moyen d'apprentissage (Causa, 2014). Les cours de français s'insèrent dans un plan d'études en portugais et anglais sur 3 ans au cours desquels la transmission et la construction progressive des concepts/ savoirs disciplinaires vient enrichir leurs compétences discursives et cognitives grâce à la transversalité et l'interdisciplinarité entre les langues et entre les contenus disciplinaires. Le développement des compétences professionnelles des étudiants en français s'appuie sur des opérations de transferts de connaissances construites dans différentes langues et dans différentes matières.

Cette démarche demande aux enseignants d'élargir leurs compétences. L'enseignant doit se confronter à un domaine qu'il connait mal car sa formation est souvent littéraire et il doit faire un effort « d'acculturation » (Lehmann, 1993 : 13). Certes il n'est pas question de se substituer aux enseignants des disciplines étudiées, mais de porter son attention sur la relation entre textes et contenus, et grâce notamment à un travail collaboratif avec les enseignants des disciplines non linguistiques et avec les professionnels sur le terrain, 
d'avoir une compréhension du fonctionnement et des postures professionnelles du secteur, de comprendre comment ce fonctionnement influence les discours et les pratiques langagières du domaine de spécialité.

L'offre, abordée sous cet angle, vise la maitrise des discours en contexte professionnel et implique l'articulation du linguistique, de différentes matières du domaine de spécialité et du mode de raisonnement et d'action professionnel. Elle sous-entend l'adaptation aux nouveaux modes de production et d'organisation du travail par la mise en place progressive de compétences permettant d'y agir et d'y interagir en posant comme premier le sens et la logique du secteur ou du métier étudié. Pour notre équipe d'enseignants à l'ESGHT, cela demande de construire et de faire évoluer nos formations sur mesure pour chaque cursus offert.

\section{L'enseignement du FLP à l'École Supérieure de Gestion, Hôtellerie et Tourisme de l'Université de l'Algarve}

24 L'École Supérieure de Gestion, Hôtellerie et Tourisme de l'Université de l'Algarve est une des Écoles Supérieures d'enseignement technique de l'Université de l'Algarve au Portugal, qui propose des Licences professionnelles, depuis 1991, et des Diplômes Technologies dans les domaines de la Gestion, de la Gestion Hôtelière et du Tourisme et, intégrées à ces formations, des unités d'enseignement en langues non généralistes.

Celles-ci ont évolué pour répondre aux changements des profils des étudiants, de la structure du secteur économique de la région et des demandes en formations. Après une présentation de l'évolution de notre offre et des facteurs internes à l'institution qui la conditionnent, nous décrirons nos choix méthodologiques (construction de programmes et pratiques de classe) motivés à la fois par l'évolution des niveaux des étudiants et par les besoins de formation technique.

\section{Une offre de langues étrangères évolutive et contextualisée}

Depuis $2000^{1}$, les licences en Tourisme, Gestion Hôtelière, Information et Animation Touristique et Secrétariat proposent, outre l'anglais qui est obligatoire pour toutes les filières, une option de langue étrangère, annuelle pendant les deux premières années et semestrielle en troisième année. C'est au niveau de cette option que les étudiants, que nous recrutons majoritairement au niveau de la région, peuvent choisir comme deuxième langue étrangère soit le français soit l'allemand.

Cette offre en langues est une offre évolutive qui dépend de la création des formations, de leur restructuration et de leur fermeture. En réponse à l'évolution de la structure économique régionale, l'ESGHT ouvre et ferme ses formations en fonction de la demande et par cette adaptation elle garantit un taux élevé d'employabilité et contribue à l'insertion professionnelle de ses diplômés.

Dans ce cadre, la prise de décision concernant l'offre de français dépend de facteurs de plusieurs ordres internes à l'institution. Ces facteurs, variables, concernent en premier lieu la politique linguistique du Conseil Scientifique et de la Direction de l'ESGHT, les 
dynamiques et les relations de pouvoir entre le départements ; ils concernent aussi les perceptions intuitives que ces différents acteurs ont des langues, de leur rôle dans les pratiques professionnelles et de l'impact économique, largement méconnu ${ }^{2}$, des compétences linguistiques sur les performances des entreprises de l'Algarve.

Indépendamment du nombre d'élèves inscrits en français dans l'enseignement préuniversitaire, le nombre de classes de français et, par conséquent, d'étudiants dépend donc d'autres types de facteurs comme le nombre d'étudiants admis chaque année dans les différents cursus, de notre capacité générale de recrutement, des tendances de recrutement des années antérieures et des perceptions du Conseil Scientifique concernant leur évolution.

Le graphique 1 représente l'évolution du nombre d'étudiants inscrits en français depuis l'année universitaire 2000/2001 à l'ESGHT.

Graphique 1. Évolution du nombre d'étudiants inscrits en langues étrangères depuis 2000

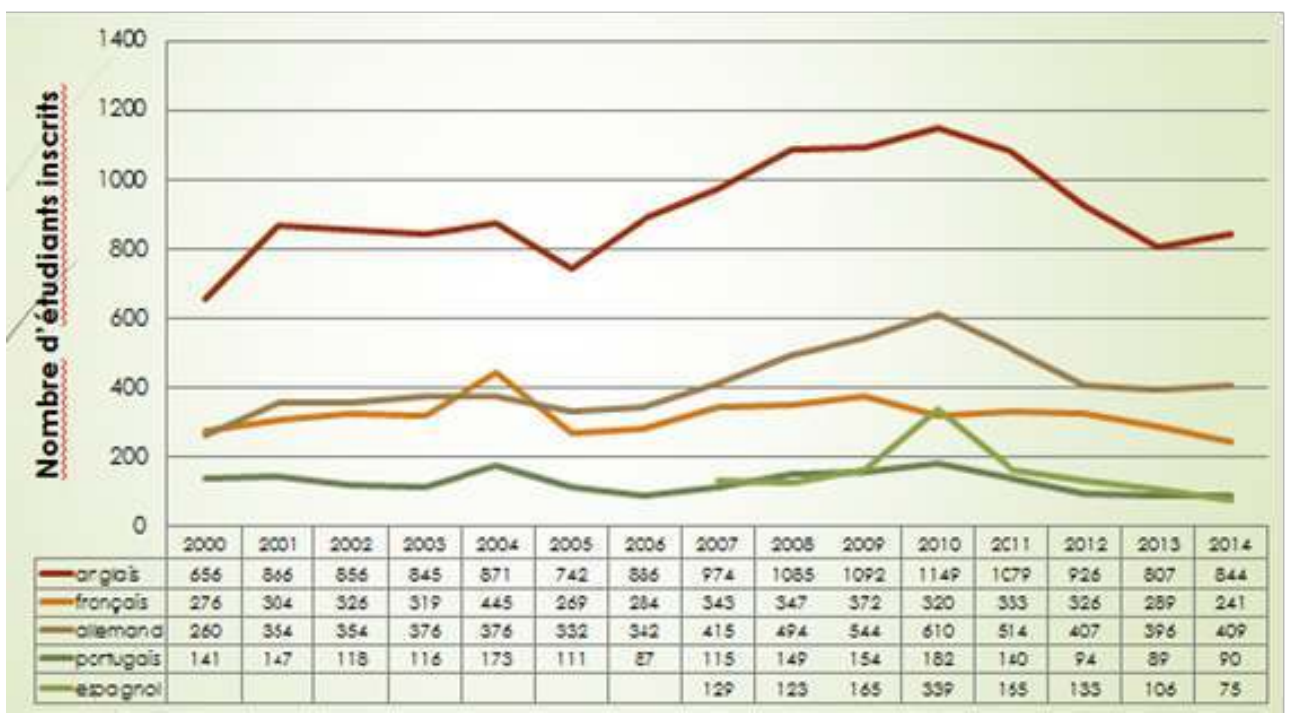

Source : Système d'information de l'ESGHT.

On peut constater qu'au long de ces 14 années, 1856 étudiants se sont inscrits en Français I et 4642 en Français I, II et III, avec un total de 241 inscrits pour l'année 2014/2015. Ces chiffres paraissent assez impressionnants mais ils doivent toutefois être relativisés par comparaison avec les données concernant les autres langues, notamment l'allemand, pour qu'on puisse leur attribuer un sens. En effet, la tendance globale au niveau de l'évolution du nombre d'étudiants inscrits en français est très semblable à celle des autres langues ${ }^{3}$. Mais, alors que le nombre d'étudiants inscrits tend à se stabiliser en allemand, en français on assiste à une légère diminution qui s'explique par l'exigence du niveau A2 en français alors que l'allemand offre l'initiation, par le recul du nombre de classes et du nombre d'années de français dans l'enseignement pré-universitaire entrainant un nombre de plus en plus élevé de candidats aux Licences en Tourisme et Gestion Hôtelière n'ayant jamais suivi de cours de français.

En effet, avant 2000, les étudiants arrivaient avec un bon niveau de français général résultat d'un minimum de 5 années d'enseignement et notre travail était essentiellement de les spécialiser aux différents domaines professionnels. Au fil des années, pour capter et 
assurer la réussite à un plus grand nombre d'étudiants, nous avons dû adapter les niveaux : le niveau d'entrée est passé de B1 à A2, au cours des années 2004/2006.

Pour l'année prochaine nous préparons donc une nouvelle étape de l'évolution de l'enseignement $\mathrm{du}$ français professionnel à l'ESGHT avec une nouvelle adaptation des niveaux offerts, c'est à dire, l'offre d'une classe d'initiation, en première année de la Licence en Tourisme car l'Espagnol est devenue la deuxième langue étrangère dans les collèges en Algarve; avec la création d'une nouvelle option d'initiation au français professionnel dans les Licences en Gestion et Marketing et l'introduction de 2 semestres de français (Oficina do Francês et Francês empresarial) dans le nouveau CTeSP (Curso Técnico Superior Profissional) de secrétariat technologique.

\section{De la construction de programmes aux pratiques de classe}

34 Considérant le niveau B1, pour certaines compétences, comme indispensable à l'interaction professionnelle dans le domaine de spécialité du Tourisme et de la Gestion Hôtelière, nous avons dû, d'abord, faire une sélection stricte des contenus, et construire des progressions thématiques, lexicales et grammaticales en fonction de leur récurrence dans les discours du domaine et à partir de l'analyse des exigences des pratiques et connaissances professionnelles. Par une démarche empirique et éclectique, nous avons adapté nos formations pour les rendre plus efficaces par le recours à des stratégies variées comme le transfert de connaissances, l'intercompréhension s'appuyant sur leurs connaissances d'autres langues et de leur domaine de spécialité qui, pour ces futurs professionnels, fait sens et constitue un facteur de motivation. Nos progressions thématiques partent, généralement, du plus spécifique, en première année, vers le moins spécifique en troisième année.

35 Ainsi, la Licence en Gestion Hôtelière, par exemple, commence par offrir un programme centré sur les situations d'accueil et de réservation, selon une démarche de type FOS et, dans le cadre du module sur la gastronomie, l'art de la table et l'accueil au restaurant, les étudiants ont 3 heures de cours pratiques où ils font, au restaurant pédagogique, la confection d'un menu français, la mise en place à la française, et une situation d'accueil et de prise de commande. En troisième année, en revanche, les étudiants doivent faire une réflexion sur la gestion de la relation client visant la résolution de plaintes et de réclamations. Ils développent et appliquent en parallèle les aspects interculturels de la relation interpersonnelle conflictuelle et l'application de ces connaissances.

36 Nous utilisons des dispositifs de formation ouverts, avec des tâches combinant plusieurs types d'objectifs, langagiers, professionnels et techniques, afin d'installer des compétences de façon plus efficace. Nous nous concentrons sur ce qui leur pose problème et adaptons les manuels, les documents et les tâches à leur réalité cognitive. Nous travaillons les quatre compétences selon la réalité de la pratique professionnelle, en privilégiant l'oral et en prenant appui sur le sens du domaine de spécialité, sur leurs intérêts, leurs projets de carrière et leurs connaissances de la réalité régionale et professionnelle. Nous prenons l'ensemble du plan d'études en considération par souci d'interdisciplinarité et avec une volonté de colmater certaines lacunes du plan d'études, nous introduisons la pratique de la relation directe avec le client, de la vente et développons la sensibilité à l'interculturel. 
Grâce à ces principes conducteurs de cohérence, de sélection et d'adaptation, la construction de compétences est accélérée et l'apprentissage gagne en efficacité, en rapidité, en autonomie et, peut-être, espérons-nous, en durabilité pour leur avenir.

\section{L'avenir de la demande de formations en français en Algarve}

La langue française au Portugal semble bénéficier en ce moment d'un regain d'intérêt dû entre autre, à la conséquence de mesures de politique fiscale du gouvernement portugais actuel, très avantageuses notamment pour les retraités français car offrant une exonération totale d'impôt sur le revenu, mais aussi pour ceux travaillant au Portugal dont les revenus sont taxés à $20 \%$ (ATA, 2012). Il leur est requis, cependant, de passer au moins 183 jours, consécutifs ou non, par an sur le sol portugais, ou bien d'y disposer d'un logement à usage d'habitation principale. Ce nouveau type de tourisme, dénommé "Tourisme fiscal », qui ne concerne pas seulement le marché français, a entraîné une augmentation des ventes immobilières à des français. Selon les données de l'APEMIP (Público, 2015), en 2014, les français ont été à l'origine de $16 \%$ des transactions immobilières étrangères au Portugal. Le Portugal est à la mode et pour la première fois, le nombre de touristes français au Portugal dépasse le nombre de touristes allemands. Cette tendance devrait s'accentuer l'année prochaine.

39 L'impact des mesures fiscales sur l'immobilier, l'augmentation de l'investissement et l'importance des recettes touristiques ont été accompagnés par les médias français et portugais et largement diffusés. Les médias portugais ont à leur tour contribué à créer chez le grand public, et chez les enseignants et les francophiles, des perceptions assez optimistes, qu'il faut nuancer, sur l'avenir de l'enseignement du français en Algarve.

Une perception favorable du grand public peut profiter au français et peut annoncer une augmentation d'élèves de français dans le secondaire. Mais ces dynamiques sont lentes et une augmentation de la demande de français dans les collèges aujourd'hui n'aura d'impacts au niveau de l'enseignement supérieur que dans six ans. Le secteur professionnel, en revanche, réagit plus rapidement et la demande de stagiaires ayant un bon niveau de français professionnel est, selon notre département de stages, en augmentation.

\section{Pour conclure}

Loin des enseignements réservés à une élite culturelle, nous avons considéré ici, selon une perspective économique, la demande de connaissances en langues envisagée sous l'angle de la compétence professionnelle et la connaissance des langues comme étant porteuse de valeur économique et comme une compétence que l'apprenant doit construire pour être rentabilisée sur le marché du travail. Cela ne signifie pas que toute référence à la littérature et à la culture soit à exclure mais elles seront sélectionnées selon un objectif spécifique en accord avec les besoins du secteur de spécialité. Ces nouvelles demandes permettent aussi, bien qu'autrement, de faciliter l'accès à la culture française, à travers, par exemple, de l'étude de la gastronomie et l'art de la table, du patrimoine culturel et touristique, par le biais de la profession. 
42 En terme de diffusion de la langue française, ce glissement du français non généraliste vers le français de spécialisé puis vers le français comme langue et compétence professionnelle entraine une augmentation des publics potentiels, précisément ceux qui n'entraient que marginalement dans les préoccupations des diffuseurs du $\mathrm{Xx}^{\mathrm{e}}$ siècle. $\mathrm{Au}$ $\mathrm{XXI}^{\mathrm{e}}$ siècle, cette demande de formation ne cesse de progresser. Ainsi le rapport Attali (2014), qui milite pour l'exploitation du potentiel économique de la francophonie à travers la promotion de la langue et de la culture françaises, présente comme proposition $\left(n^{\circ} 7 / 53\right)$ : “Développer l'offre d'apprentissage du français professionnel au sein des instituts français, des alliances françaises et des groupes français privés" notamment à travers l'offre de formation en français professionnel dans les secteurs juridique, médical, touristique et de l'hôtellerie.

La diffusion de la langue française au Portugal doit tirer parti de cette tendance et de cette récente perception favorable au français. Il nous semble essentiel de mener une action engagée au niveau des institutions où nous travaillons pour proposer des formations de différents types adaptés aux besoins diversifiés des nouveaux publics régionaux. Mais pour mener cette proposition à bon port, il faut valoriser l'utilisation contemporaine, utile et rentable du français dans les activités professionnelles. Il s'agit pour les enseignants de se former aux domaines techniques et, dans le cadre de nos institutions universitaires, de proposer des unités d'enseignement dans les Licences et les Diplômes technologiques, en justifiant la pertinence et l'adéquation de ces formations en langues par des résultats de recherches sur les impacts économiques de l'utilisation de ces compétences linguistiques.

\section{BIBLIOGRAPHIE}

ALLC (Australian Language and Literacy Council) (1994). Speaking of business. The needs of business and industry for language skills. Canberra : National Board of Employment, Education and Training. ATA (Autoridade Tributária e Aduaneira) (2012). Regime fiscal dos Residentes não habituais. Circular nº9/2012 de 3 de Agosto de 2012.

ATTALI, Jacques (2014). La Francophonie et la francophilie, moteurs de croissance durable. Paris : La documentation française.

BENSFIA, Abdellatif, MABrour, Abdelouahed et MGHARFAOUI Khalil (2013). "L'impact du choix de la langue sur les débouchés et carrières professionnels : points de vue d'étudiants”, in Recherches en didactique des langues et des cultures : Les Cahiers de l'Acedle, volume 10, ํㅡㄴ, 2013.

BOERS, Frank \& LINDSTROMBERG Seth (2006). “Cognitive linguistic applications in second or foreign language instruction: rationale, proposals, and evaluation" in KRISTIANSEN, G., ACHARD, M., DIRVEN, R., \& RUIZ DE MENDOZA IBÁÑEZ, F.J. (Eds.) Cognitive linguistics : Current applications and future perspectives. Berlin/New York : Mouton de Gruyter, pp. 305-355.

CAUSA, Mariella (2014), « Compétence discursive et enseignement d'une discipline non linguistique : définition, diversification et pratiques formatives » in Les Carnets du Cediscor, [En 
ligne], 12 | 2014, document 6, mis en ligne le 01 mars 2016.

<URL: http://cediscor.revues.org/964>

COMMISSION EUROPEENNE (2006). ELAN: Effects on the European Economy of Shortages of Foreign Language Skills in Enterprise. The National Centre for Languages (CILT), [disponible le 1/12/2014]

<URL: https://www.llas.ac.uk/news/2772>

DE RYCKER, Antoon, \& DE KNOP, Sabine (2015). Integrating Cognitive Linguistics and Foreign Language

Teaching - Historical Background and New Developments. Journal of Modern Languages, 19(1).

EVANS, Vyvyan (2014). The language myth: uncovering the true nature of language, mind and being

human. Cambridge: Cambridge University Press.

FAUCONNIER Gilles et TURNER, Mark (2008). The way we think: Conceptual blending and the mind's hidden complexities. New York: Basic Books.

FUCHS, Catherine (2008). "Linguistique française et cognition”, in: DURAND, J. HABERT, B., LAKS B.

(éds.) Congrès Mondial de Linguistique Française CMLF’08. EDP Sciences, 61-72.

GRIN, François, SFREDDO, Claudio et VAILLANCOURT, François (2009). Langues étrangères dans l'activité professionnelle. Rapport final de recherche. Genève : Université de Genève.

LEHMANN, Denis (1993). Objectifs spécifiques en langue étrangère. Paris: Hachette.

LITTLEMORE, J. (2009). Applying cognitive linguistics to second language learning and teaching. Springer.

MANGIANTE, Jean-Michel et PARPETTE, Chantal (2004). Le Français sur objectif spécifique : de l'analyse de besoins à l'élaboration d'un cours. Paris : Hachette.

MANGIANTE, Jean-Michel et PARPETTE, Chantal (2011). Le Français sur objectif universitaire. Paris :

P.U.G.

MOURLHON-DALLIES, Florence (2008). Enseigner une langue à des fins professionnelles. Paris : Didier.

PUREN, Christian (2005). » Domaines de la didactique des langues-cultures. Entrées libres » in Les Cahiers pédagogiques $n^{\circ} 437$, novembre 2005, Paris, CRAP pp. 41-44. Paris.

PUREN, Christian (2006). "De l'approche communicative à la perspective actionnelle", in Le Français dans le Monde $\mathrm{n}^{\circ}$ 347, Paris : FIPF-CLE international, pp. 37-40.

RICHIER, Jean-Jacques (2008). « Le F.O.S. : une didactique du langage et de l'action », Points Communs, mai 2008, $\mathrm{n}^{\circ} 34$, pp. 7-10.

RICHIER, Jean-Jacques (2014). « Conditions d'une mise en œuvre de la perspective actionnelle en didactique des langues ", in Recherche et pratiques pédagogiques en langues de spécialité, vol. XXXIII, pp. 33-49.

SOARES, Rosa (2015). « Franceses aproximam-se dos chineses na compra de imóveis em Portugal » [on-line] PÚBLICO, Edition du 10 février 2015 [disponible le 10/02/2015]

<URL : http://www.publico.pt/economia/noticia/francesas-aproximamse-dos-chineses-nacompra-de-imoveis-em-portugal-1685577 >

SowA, Magdalena (2014). «FS, FOS, FLP... Étiquettes vides ou concepts éducatifs opérationnels?» in Le FOS aujourd'hui : quel périmètre et quelle influence en didactique des langues? Jean-Marc MANGIANTE et Jean-Jacques RICHER (Coord.) Points Communs - Recherche en didactique des langues sur objectif(s) spécifique(s), n 1, pp. 18-28. 
VOLLMER, Helmut (2009). Langue(s) des autres disciplines. Conseil de l'Europe, disponible sur la Plateforme de ressources et de références pour l'éducation plurilingue et interculturelle : <URL : http://www.coe.int/t/dg4/linguistic/langeduc/BoxD2OtherSub_fr.asp\#s1>

\section{NOTES}

*. http://www.mosalingua.com/blog/2009/04/26/7-bonnes-raisons-dapprendre-une-langueetrangere/

1. Le Système d'Information de l'Intranet de l'ESGHT dispose de données seulement à partir de cette date.

2. La recherche dans ce domaine nous semble essentielle pour la description de l'évolution de l'enseignement des langues professionnelles au Portugal mais aussi pour la production de données destinées à soutenir et motiver les choix des langues offertes dans un cursus.

3. On peut constater à partir de 2004/2005 l'impact du passage des licences de 4 à 3 ans, en raison de l'adaptation aux Accords de Boulogne, processus au cours duquel la 2ème langue a perdu entre 1 et 3 semestres par licence.

\section{RÉSUMÉS}

Dans le cadre de la diffusion du français, le français comme langue professionnelle (FLP) représente une demande croissante. Le FLP est valorisé sur le marché du travail car les nouvelles mobilités et les changements d'organisation de la production ont transformé les pratiques langagières en pratiques professionnelles. Il convient d'adapter les offres de formation à ces demandes spécifiques et évolutives. Nous témoignons de notre participation à cette diffusion au sein d'une institution supérieure en Algarve. Depuis 1992, nous adaptons nos formations aux besoins des étudiants et des secteurs d'activité régionaux dont la logique professionnelle est au cœur de nos dispositifs d'enseignement et de notre didactique intégrée. De récentes mesures fiscales ont engendré une augmentation de la demande française sur les marchés du tourisme et de l'immobilier portugais. Il s'agit donc de saisir cette opportunité, pour monter des formations répondant aux exigences de ces secteurs et de participer ainsi à la diffusion de la culture française.

The demand of French as a professional language (FLP) is growing. Professional language skills is valued on the labor market due to new forms of mobility and changes in the organization of production that has transformed language practices into professional practices. Syllabus and training should be adapted to these specific and changing demands. Here we present the testimony of our participation in this process at the University of the Algarve. Since 1992, we have been adapting our courses to the needs of our students and to the needs of the regional economic sectors whose professional logic is at the core of our teaching strategies and practices. Recent fiscal measures have led to an increase in the demand from French nationals in the Portuguese tourism and real estate markets. We should therefore use these opportunities to develop training courses in FLP that meet the requirements of these sectors; this in turn will allow us to play an active part in the diffusion of French culture. 
INDEX

Mots-clés : formation en français professionnel, méthodologie, diffusion, tourisme, Portugal

Keywords : training in professional French, methodology, dissemination, tourism, Portugal

\section{AUTEURS}

\section{CATHERINE PASQUIER}

Université de l'Algarve - ESGHT

cpasquie[at]ualg.pt

\section{IDÍLIA DOS SANTOS}

Université de l'Algarve - ESGHT

ibarros[at]ualg.pt 